\title{
Un modelo de afiliación informal: prácticas socioeducativas de estudiantes de la Sede Interuniversitaria de Alajuela
}

\author{
Cavallini Solano, Hennia María \\ Alajuela \\ Revista Educación, vol. 42, núm. 2, 2018 \\ Universidad de Costa Rica, Costa Rica \\ Disponible en: http://www.redalyc.org/articulo.oa?id=44055139015 \\ DOI: https://doi.org/10.15517/revedu.v42i2.25832
}

Un modelo de afiliación informal: prácticas socioeducativas de estudiantes de la Sede Interuniversitaria de

Esta obra está bajo una Licencia Creative Commons Atribución-NoComercial-SinDerivar 3.0 Internacional. 


\title{
Un modelo de afiliación informal: prácticas socioeducativas de estudiantes de la Sede Interuniversitaria de Alajuela
}

\author{
Hennia Maria Cavallini Solano [1] \\ Universidad de Costa Rica, Costa Rica \\ (D) http://orcid.org/0000-0003-3907-2397
}

\author{
DOI: https://doi.org/10.15517/revedu.v42i2.25832 \\ Redalyc: http://www.redalyc.org/articulo.oa?id=44055139015
}

\section{Resumen:}

Este artículo es el producto generado de una de las dimensiones de análisis de la investigación denominada "Las capacidades de las personas de la comunidad que orientan la formulación del currículum de la carrera de ingeniería mecánica con énfasis en protección de incendios de la sede interuniversitaria de Alajuela”. Se busca develar el modelo de afiliación adoptado por la población de estudiantes, herramienta que servirá de base para la reformulación del currículo de la carrera de Ingeniería Mecánica con Énfasis en Protección de incendios. Este modelo se asocia a la capacidad de afiliación y se devela como práctica socioeducativa. El abordaje metodológico de este trabajo es de corte mixto, en el que se utilizaron diferentes estrategias de observación y entrevistas, que permitieron abordar, por medio de un sistema de votación y el programa Net Draw, la construcción gráfica de mapas de afiliación. Los principales hallazgos refieren a la caracterización de un modelo de afiliación informal de encadenamiento horizontal de corto plazo, dependiente de la zona espaciamiento.

Palabras Clave: Afiliación informal, prácticas socioeducativas, currículo, ingeniería.

\section{ABstract:}

This article is the generated output from one of the research dimensions of analysis called " Capabilities of the community individuals that guide the curriculum formulation of the Mechanical Engineering major with emphasis on fire protection at Sede Interuniversitaria de Alajuela (Alajuela's Inter- university branch)." This article aims at unveiling the affiliate university model adopted by the student population, tool serving as a basis for the Mechanical Engineering curriculum reformulation with emphasis on fire protection. This model of affiliation is associated with the capacity of affiliation and it is considered a socio-educational practice. The methodological approach of study was mixed and used different observation strategies and interviews allowing voting by means of a voting system as well as the NetDraw software for the construction of graphical affiliation maps. The main findings are related to an informal characterization short-term horizontal chaining model dependent on the spacing area.

KEYWORDS: Informal affiliation, socieducational practices, curriculum, engineering.

\section{INTRODUCCIÓN}

El presente trabajo se realiza en el marco de la investigación denominada: "Las capacidades de la comunidad que orientan la formulación del currículum de la carrera de Ingeniería Mecánica en la Sede Interuniversitaria de Alajuela”, en donde se consideran las seis capacidades humanas, denominadas: 1) sentidos e imaginación, 2) pensamiento y razón, 3) emociones, 4) control del entorno, 5) vida salud e integridad corporal y 6) afiliación.

La afiliación es la capacidad humana en la que se centrará este artículo. Esta es una dimensión que se asocia a algunas prácticas socioeducativas que reproduce el estudiantado de la carrera de Ingeniería Mecánica con

\section{NotAS DE AUTOR}

[1] Licenciada en Ingeniería Mecánica de la Universidad de Costa Rica, con tres maestrías: una en procesos de manufactura de la Universidad de Costa Rica (2007) y dos maestrías obtenidas en la Universidad de Navarra-Colegio de Ingenieros industriales, una en Metalurgia Física y otra en Ciencia de los Materiales (1994). Ingeniera de proceso y calidad de la compañía ALUNASA S.A (1994-1997), directora de la Escuela de Ingeniería Mecánica de la Universidad de Costa Rica (2004-2012). Coordinadora General de la Sede Interuniversitaria de Alajuela (2011-2013). Coordinadora de la Unidad de Vida Estudiantil de la Sede Interuniversitaria de Alajuela del 2012-2015. Coordinadora de la Carrea de Ingeniería Mecánica con Énfasis en Protección contra Incendios del 2011-actualmente. profesora de diferentes cursos de la carrera de Ingeniería Mecánica. Par evaluadora de las carreras de Ingeniería Mecánica de Universidades Centroamericanas y Cuba. 
Énfasis en Protección de Incendios de la Sede Interuniversitaria de Alajuela, en el salón de clase y que dan origen a un modelo de afiliación informal.

El propósito de este artículo es el de obtener una representación del modelo de afiliación, su descripción y la forma en que este se estructura. Esto permitirá, al personal docente tomar decisiones en cuanto a rutas metodológicas a seguir en su salón de clase. En síntesis, se tratará de representar los procesos por medio de los cuales el estudiantado de la carrera de Ingeniería Mecánica con Énfasis en Protección de Incendios interacciona con sus pares en el salón de clase, herramienta básica e indispensable en la formulación del currículo de en una carrera.

Ahora bien, con respecto a la conformación de este artículo, estará compuesto por cuatro apartados. En primer lugar, el referente teórico que comprende los temas de teoría de las capacidades, prácticas socioeducativas y los modelos de afiliación. En segundo lugar, la metodología de corte mixto se desarrolla con base en técnicas de observación y entrevistas. En tercer lugar, el análisis de resultados, en donde se trabaja con el programa "NETDRAW", el cual permite analizar, de forma cuantitativa y gráfica, el comportamiento de la red social generada por el estudiantado durante el proceso de recolección de datos y, en cuarto lugar, el análisis de fragmentos textuales de observaciones de campo y entrevistas a docentes. Entre los factores más importantes que se destacan en esta investigación se encuentra la caracterización de los modelos de afiliación que se dan en la población estudiada, modelos que ayudarán a definir las estratégicas didácticas de trabajo en clase, las cuales, como se mencionó anteriormente, se pueden considerar como una de las bases fundamentales para la reformulación curricular.

\section{Referente teórico}

\section{Teoría de las capacidades}

En esta investigación se asume como referente la teoría de las capacidades desarrollada por Sen (1999) y Nussbaum (1992), los cuales abordan la teoría de las necesidades, que habilitan el término capacidad y deshabilitan el término necesidad.

Tanto Sen (1999) como Nussbaum (2005), en la teoría de las capacidades, destacan al ser humano como único, para lo cual se basan en su situación real, al considerar que su calidad de vida depende de los contextos específicos de donde proviene, de los recursos que posee, que puede aprovechar y que se pueden visualizar como sociales, culturales, políticos y económicos. Recursos que contribuyen en la conformación del "habitus" ${ }^{[2]}$ y del "campus" [3], conceptos que explican las prácticas sociales y culturales de la familia como unidad de vida base.

En la teoría de las capacidades, Sen (1999) propone una lista de dieciséis capacidades o funcionamientos centrales, las cuales abarcan desde los estados y actividades más elementales, a las actividades superiores de la persona, a saber, ser capaz de estar sana, ver, evitar el hambre, ayunar, tener vivienda, tener seguridad física, evitar ser agredida físicamente, trasladarse sin peligro, tener ropa, evitar vergüenza por la ropa que lleva, estar junto a los seres queridos, afiliación, vivir sin estar estigmatizada, llevar una vida normal actuar con sensatez, estar equilibrada y sentirse feliz (Cejudo, 2006). De acuerdo con Gough (2007), estas capacidades se refieren al conjunto de funcionamientos que hacen que el ser humano se pueda desenvolver cotidianamente.

Por otro lado, Nussbaum (1992) identifica y define las que denomina diez esferas de experiencias humanas o capacidades de funcionamientos centrales humanos: vida, salud, integridad corporal, afiliación, emociones, control del entorno, sentidos-imaginación y pensamiento, razón práctica, capacidad para jugar y otras especies.

No obstante, las capacidades o funcionamientos centrales constituyen recursos que tiene el sujeto en su poder (Sen, 1981). Estas se pueden definir como la facultad que tiene cada individuo para poder lograr 
efectivamente algo, lo que posee y se posibilita para ser, hacer y estar. Además, se pueden estimular o desarrollar por medio de prácticas socioeducativas.

\section{Prácticas socioeducativas}

Las prácticas socioeducativas que propician el desarrollo de las capacidades en una comunidad se basan en los recursos culturales y sociales de la familia. Esta marca las diferencias en la formación de su prole y, de acuerdo con Marchesi (2000), es la que establece las pautas de cómo sus miembros se comuniquen, del nivel de lenguaje que manejen, de qué información intercambien, de su participación en actividades culturales, de cómo realicen el seguimiento de los estudios, de los libros que leen y de la información que intercambien.

Es así como los recursos culturales y sociales con que cuenta cada familia conforman el "habitus" de la prole, el cual se transforma dinámicamente a lo largo del tiempo (Bourdieu, 1991), especialmente cuando el individuo inicia un proceso de afiliación al sistema formal educativo (primaria, secundaria y educación superior), que se considerará de ahora en adelante como una comunidad secundaria.

A las comunidades secundarias se afilian actores como personal docente, administrativo y técnico, agentes socioeducativos, con recursos culturales y sociales, diferentes, los cuales conformarán un nuevo "campus", el cual será fundamental para la generación de los símbolos y códigos que darán origen a un sistema de comunicación propio de un grupo específico y afín. Cabe destacar que no depende de la cantidad de recursos sociales y culturales que posee el individuo, sino de la forma en que estos se vayan a transmitir (Marchesi, 2000). Por otro lado, la forma en que se transmiten estos recursos depende directamente de los procesos educativos (informal y formal) en los que está inmerso el individuo y son el soporte de sus fases de formación. Establecen sus capacidades de afiliación, de adaptación y de transformación en su entorno social y, según Zamudio (2011), son las que dan origen a prácticas socioeducativas que se pueden asociar directamente a un modelo de afiliación.

\section{Modelos de afiliación}

Los modelos de afiliación buscan describir las estrategias que los individuos utilizan para enfrentar los diferentes contextos o entornos sociales a los que se deben integrar en su proceso de formación, estos van de la mano de los procesos de socialización, en donde se transmiten al individuo valores, representaciones y prácticas sociales, que les permiten formar parte de un grupo o grupos sociales, de una comunidad, de una asociación o de una institución.

En este trabajo se toman como base para clasificar los modelos de afiliación, las propuestas de modelos de asociatividad formal e informal de Lozano (2010) y Telle (2004). Estos autores caracterizan el modelo de asociatividad formal como una organización con requisitos, normativas y credenciales de pertenencia, y, al modelo de asociatividad informal, como una organización sin andamiaje, que funciona por costumbre y tradición.

\section{Metodología}

Esta investigación se realizó a lo largo del primer semestre del 2015, en la Sede Interuniversitaria de Alajuela para una población de veinte estudiantes del curso de mecánica 1, IM-0207 (Mecánica1-Estática) de las carreras de ingeniería y se aborda metodológicamente con un enfoque mixto, descriptivo, exploratorio y aplicado. Descriptivo y exploratorio porque se indagan las prácticas socioeducativas de estudiantes que son procedentes exclusivamente de la provincia de Alajuela; y aplicado, ya que por medio de los hallazgos 
obtenidos en el proceso de indagación, se podrán representar los modelos de afiliación con los que se caracterizará la población estudiada.

Además, esta investigación responde a dos perspectivas teóricas : a) la teoría crítica en su dimensión sociocrítica, dimensión que se refiere a la emancipación humana personal y social (Aronowits y Giroux, 1985), y que es el fundamento para esta investigación, ya que se pretende buscar que el sujeto tome conciencia del papel protagónico que tiene que asumir dentro de su comunidad al establecer, potenciar y fortalecer la importancia y riqueza del desarrollo de distintas capacidades, conocimientos, habilidades y destrezas, para llegar a una transformación social, política, económica, cultural, crítica y reflexiva, b) la teoría interpretativa -dimensión interaccionismo simbólico, dimensión que, según Crotty (1998) y Former y Latorre (1996), explora las comprensiones de la cultura al defender que la experiencia humana está mediatizada por la interpretación que los individuos realizan en la interacción con el mundo social y se acoge a esta dimensión, ya que la sociedad es interacción, un conjunto de reacciones comunes de los individuos, que conducen a un conjunto de rasgos culturales que se deben interpretar y definir para identificar las capacidades de la unidad social por estudiar.

Por otro lado, el desarrollo del proceso de investigación se basó en entrevistas semiestructuradas a grupos focales, estrategias necesarias para obtener la perspectiva interna de los actores y la observación participante, fundamental para la descripción de las unidades sociales y escenas culturales a través de la vivencia de las experiencias de los actores involucrados. Esta decisión se toma debido al carácter mixto de la investigación y a la forma en que estas estrategias de obtención de la información favorecen la interacción social con los sujetos del escenario; además, permiten mantener la capacidad de respuesta y adaptabilidad a las circunstancias. En el proceso de recolección de datos, para este caso, según Latorre, Del Rincón \& Arnal (1997), por ser un modelo cíclico tipo espiral, las estrategias podrán ser utilizadas como considere apropiado quien investiga.

\section{Observación participante}

Se trabaja con observación grupal de veinte estudiantes del curso de mecánica 1, IM-207 (Estática), ocho pertenecían a la carrera de Ingeniería Mecánica con Énfasis en Protección Contra Incendios y doce a la carrera de Ingeniería Industrial. Del grupo de veinte estudiantes solo cuatro son de sexo femenino y pertenecen a la carrera de Ingeniería Industrial, el promedio de edad es de veintiún años.

El proceso de observación se realizó durante un periodo de ocho semanas, en este proceso se pretende observar la forma en cómo el estudiantado se afilia en su salón de clase cuando trabaja de forma grupal, para esto se utiliza una ficha de observación estructurada en cuatro secciones:

- Primera sección: en esta se indica el día, la hora, de inicio la finalización, número de estudiantes.

Segunda sección: Ubicación de estudiantes por puesto en el salón de clase.

Tercera sección: Descripción de las actividades individuales, rúbrica de participación.

Cuarta sección: Descripción de las actividades grupales, tabla de conformación de grupos, y esquema de interacciones grupales.

\section{Entrevistas}

Para las entrevistas semiestructuradas, se trabajó con cinco docentes, con estas entrevistas se busca propiciar un encuentro cara a cara con docentes que trabajen con estudiantes de la carrera de Ingeniería Mecánica con Énfasis en Protección de Incendios, de forma que se pueda obtener información acerca de la población en estudio, para poder articular con la información obtenida en los episodios de observación. Para estas entrevistas se consideró:

- Una sola persona investigadora y una sola entrevistada. 
Un solo encuentro para cada entrevista.

Un guión preestablecido, y algunas preguntas semiestructuradas.

Una introducción que se leyó a cada persona entrevistada antes de iniciar la entrevista en donde se explicó en qué consistió la investigación.

Se realizaron cinco entrevistas a docentes.

Todas las entrevistas se grabaron con autorización de las personas entrevistadas y posteriormente se transcribieron.

\section{ObSERVACión DEL TRABAJO GRUPAL EN EL SALÓN DE CLASE}

Para el proceso de observación en el salón de clase, se planean dos métodos específicos para el trabajo grupal. El primero se aplica las ocho semanas del proceso de observación y consiste en la formación libre de grupos de trabajo y el personal docente da la liberta a sus estudiantes de que se organicen según su propio criterio. El segundo método se aplica en las dos últimas semanas de observación, en este el personal docente conforma los grupos de trabajo, selecciona a una persona que coordine y da instrucciones para desarrollar los problemas o tópicos a estudiar.

Primer método de trabajo grupal: Para este método, el personal docente solicitó a sus estudiantes la formación, libre, de cinco grupos o equipos de trabajo, compuestos por cuatro estudiantes, identificados como, equipo rojo, equipo amarillo, equipo verde, equipo azul, equipo naranja; el estudiantado organizan solo su trabajo (ver Figura 1).

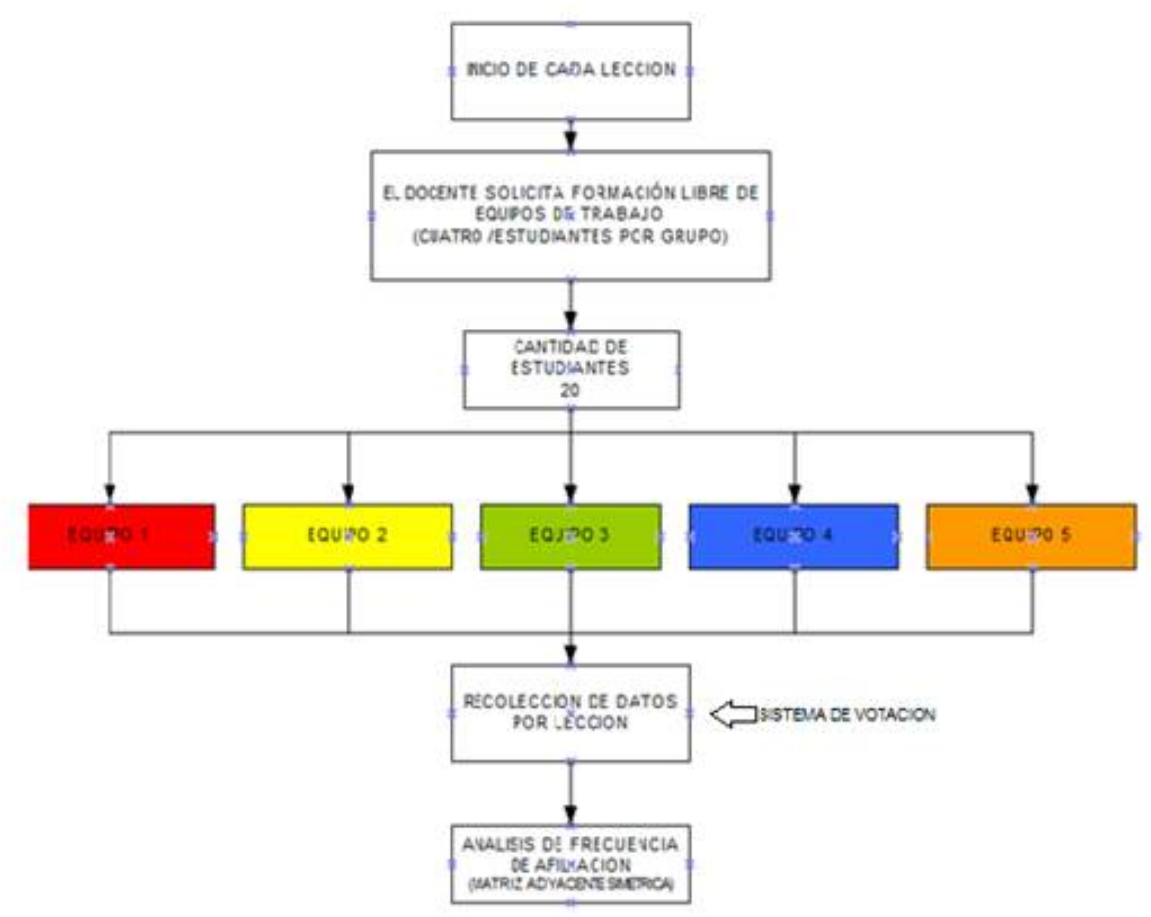

FIGURA 1.

Proceso mapeo de afiliación.

Elaboración propia de la autora (2015).

Una vez conformados los grupos de trabajo, el personal docente inicia la lección, en la cual el estudiantado debe ir dando sus respuestas a las preguntas o problemas que se planteen. Estas respuestas son transmitidas a un receptor, en donde se almacenan los datos emitidos por cada estudiante. En este sentido, como se mencionó anteriormente, los datos responden a las respuestas emitidas por el estudiantado, mediante un 
dispositivo de voto y receptor. Ahora bien, a cada estudiante se le entregó un dispositivo de voto, con un código de identificación, consecuentemente todas las participaciones estudiantiles quedan así almacenadas automáticamente en la computadora o receptor que utilice el personal docente, se genera así a una base de datos, este proceso se muestra en la Figura 2.

Para el análisis de datos obtenidos en el proceso de observación del trabajo grupal, primer método, se desarrolla con un sistema de mapeo denominado "mapa de afiliación", el cual se basa en una matriz adyacente simétrica, la que fue alimentada con los datos obtenidos durante el proceso de observación, por medio del sistema de votación. De esta forma se representan las relaciones binarias existentes entre los datos. Lo anterior facilita la generación de grafos, que permiten una caracterización sencilla de los modelos de afiliación que se gestan en la población .

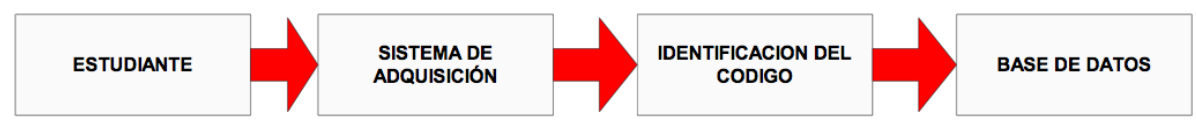

Figura 2. Proceso de adquisición de datos por medio del sistema de votación. Elaboración propia de la autora (2015).

Terminado el periodo de recolección de datos se trabaja con la matriz simétrica adyacente, con la que se determinan las frecuencias de afiliación, las cuales se generan la red de asociación general e individual de los individuos, por medio del paquete NetDraw, software, que permite visualizar las redes de trabajo o redes de comunicación y de un análisis numérico, de la población en estudio.

Segundo método de trabajo grupal: en este método el personal docente organiza los grupos, nombra a una persona coordinadora y da las instrucciones a seguir para el desarrollo del trabajo grupal. El proceso de recolección de datos se lleva a cabo mediante la ficha de observación.

Finalmente, una vez que se concluye el proceso de recolección de datos, se inicia el proceso de interpretación de resultados, articulando los datos obtenidos por medio de las entrevistas, y las notas en el periodo de observación.

\section{RESUltados y ANÁLISIS}

\section{Mapas de afiliación}

Para el análisis de resultados se cuenta con una matriz simétrica adyacente, que se presenta en la Figura 3, con esta matriz se obtiene la frecuencia con la que se afilia el estudiantado durante el periodo de observación. 


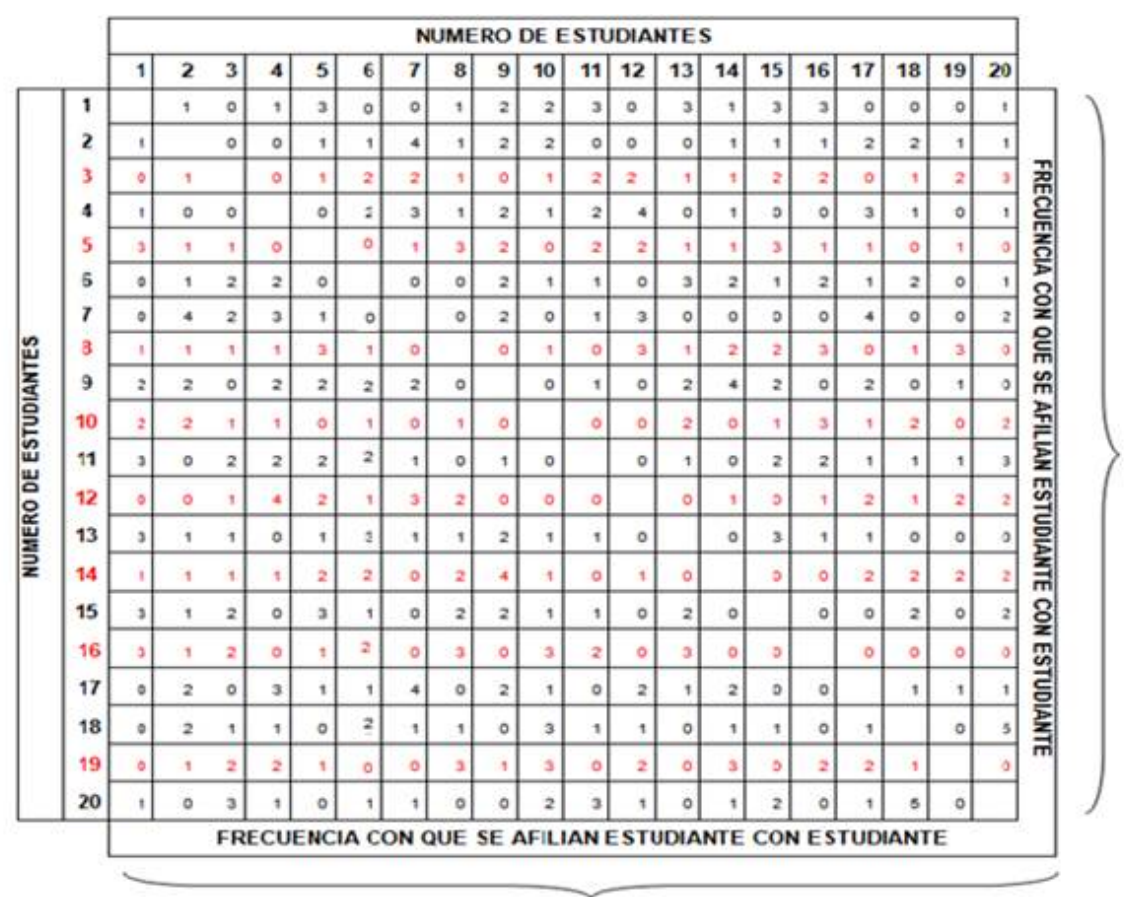

\section{FIGURA 3}

Figura 3. Matriz simétrica adyacente Elaboración propia de la autora[A2] (2015)

Se facilita así la construcción del mapa de afiliación correspondiente al periodo de observación de la población estudiada, el cual se observa en la Figura 4. Cada punto en color representa a cada estudiante y en cada línea de unión entre puntos aparecen las frecuencias de afiliación para cada estudiante.

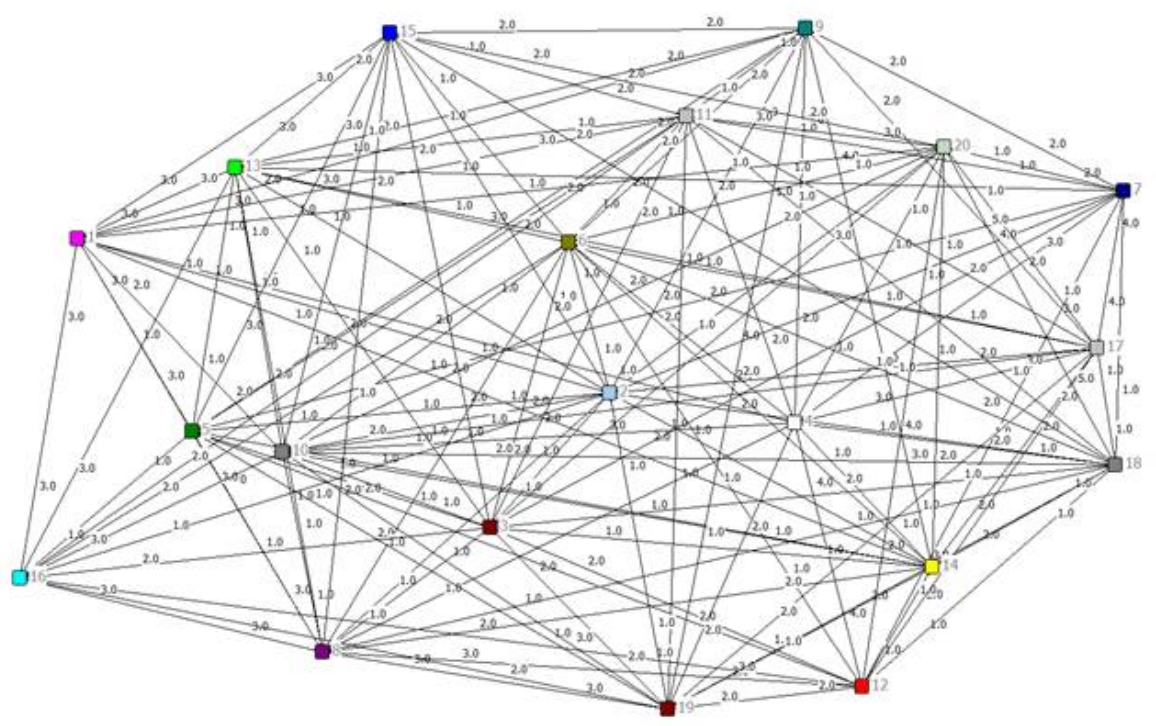

Figura 4. Mapa de afiliación generado por estudiantes durante el periodo de observación, basado en la matriz de frecuencias. Elaboración propia de la autora (2015).

Además del mapa de afiliación general, se obtuvo para cada estudiante un mapa individual, con el cual se puede determinar el número de estudiantes, con los cuales este no se afilió durante el periodo de observación, un ejemplo de este tipo de mapa se puede ver en la Figura 5. 


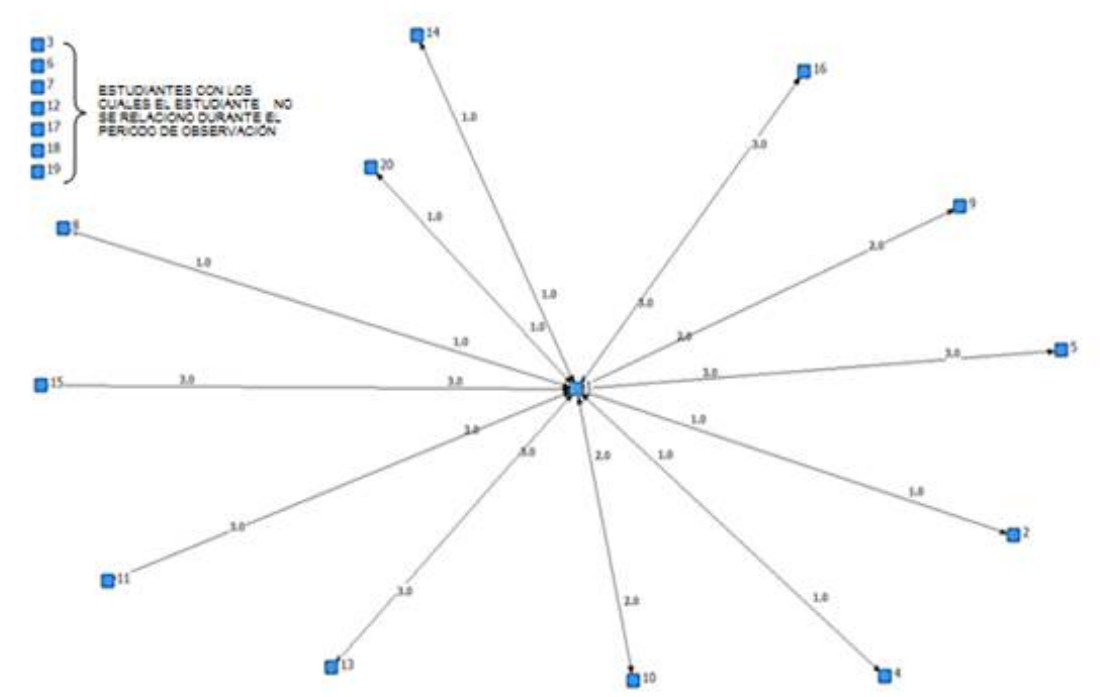

Figura 5. Mapa de afiliación individual, estudiante 1, generado durante el periodo de observación. Elaboración propia de la autora (2015).

En la esquina superior derecha de la Figura 5, se pueden observar estudiantes con los que el estudiante número uno no se afilió durante el periodo de observación. De esta misma forma se presenta cada uno de los mapas individuales por estudiante.

Por lo tanto, con la información obtenida y haciendo un análisis de los mapas individuales se construye la Tabla 1 que facilita la construcción del gráfico de la Figura 6, el cual permite observar con mayor claridad la diferencia de frecuencias de afiliación, por sexo de estudiante y la carrera a la que pertenece. 
TABLA 1

Estudiantes con quienes no hay afiliación durante el periodo de observación

\begin{tabular}{|c|c|c|c|c|c|c|c|c|c|c|c|}
\hline \multirow{2}{*}{$\begin{array}{l}\text { ESTUDIANTE } \\
1 \\
\end{array}$} & \multirow{2}{*}{$\begin{array}{c}\text { SEXO } \\
\text { F/FEMENINO } \\
\text { M/MASCULINO } \\
M\end{array}$} & \multirow{2}{*}{$\begin{array}{c}\text { CARRERA } \\
\text { H/NINGENIERIA INDUSTRIAL } \\
\text { IMUNGENIERIA NECANICA } \\
\text { II }\end{array}$} & \multicolumn{8}{|c|}{$\begin{array}{l}\text { ESTUDIANTES CON LOS QUE NO HAY AFIUIACION A } \\
\text { LO LARGO DEL PERIODO DE OBSERVACION }\end{array}$} & \multirow{2}{*}{$\begin{array}{l}\text { NUMERO DE NO AFILIACIONES } \\
\text { EN PERICDO DE OBSERVACION } \\
7\end{array}$} \\
\hline & & & 3 & \multirow{2}{*}{$\frac{6}{11}$} & \multirow{2}{*}{$\frac{7}{12}$} & \multirow{2}{*}{12} & \multirow{2}{*}{17} & \multirow{2}{*}{18} & \multicolumn{2}{|l|}{19} & \\
\hline 2 & $M$ & II & 4 & & & & & & & & 3 \\
\hline 3 & $M$ & $\mathrm{IM}$ & 1 & 4 & 9 & 17 & & & & & 4 \\
\hline 5 & $M$ & IM & 4 & 6 & 10 & 18 & 20 & & & & 5 \\
\hline 6 & $M$ & II & 1 & 5 & 6 & 7 & 15 & & & & 5 \\
\hline 7 & $\mathrm{~F}$ & II & 1 & 6 & 8 & 10 & 14 & 15 & 16 & 19 & 8 \\
\hline 8 & $M$ & $\mathrm{IM}$ & 7 & 9 & 11 & 17 & 20 & & & & 5 \\
\hline 12 & $M$ & $\mathrm{IM}$ & 2 & 8 & 10 & 12 & 14 & & & & 5 \\
\hline 13 & $M$ & II & 4 & 12 & 14 & 18 & 15 & 20 & & & 6 \\
\hline 14 & $M$ & $\mathrm{IM}$ & 7 & 11 & 13 & 15 & 16 & & & & 5 \\
\hline 15 & $M$ & II & 4 & 7 & 12 & 14 & 16 & 17 & 19 & & 7 \\
\hline 16 & $M$ & $\mathrm{IM}$ & 4 & 7 & 9 & 14 & 15 & 17 & 18 & 20 & 8 \\
\hline 17 & $\mathrm{~F}$ & II & 1 & 3 & 8 & 11 & 15 & & & & 5 \\
\hline 18 & $M$ & II & 1 & 5 & 9 & 13 & 16 & & & & 5 \\
\hline 19 & $\mathrm{M}$ & $\mathrm{IM}$ & 1 & 6 & 7 & 13 & 15 & 20 & & & 6 \\
\hline 20 & $\mathrm{M}$ & II & 5 & 8 & 9 & 13 & 16 & 19 & & & 6 \\
\hline
\end{tabular}

Elaboración propia de la autora (2015).

Basada en los resultados obtenidos se construye la Figura 6, en la que se puede observar que estudiantes que se afiliaron con menos frecuencia fueron, en primer lugar las estudiantes, de la carrera de Ingeniería Industrial; en segundo lugar los estudiantes de la carrera de Ingeniería Industrial, y en tercero y último lugar, los estudiantes de la carrera de Ingeniería Mecánica con Énfasis en Protección de Incendios.

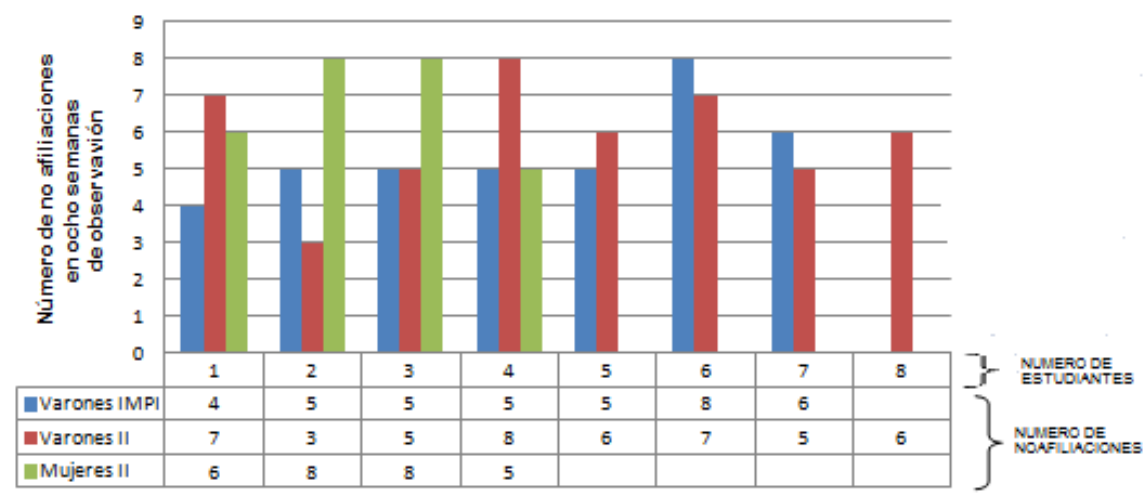

FIGURA 6

Figura 6. Número de no afiliaciones de estudiantes en ocho semanas de observación. Elaboración propia de la autora (2015).

Al hacer un análisis detallado por carrera, el mayor promedio de no afiliación por carrera corresponde a la carrera de Ingeniería Industrial. Sin embargo, si se analiza por sexo y considerando que no hay estudiantes del sexo femenino en esta población procedentes de la carrera de Ingeniería Mecánica con Énfasis en Protección de Incendios, los promedios de no afiliaciones entre estudiantes del sexo masculino se aproximan mucho. Quiere decir que las estudiantes del sexo femenino son las que, probablemente, marquen la diferencia en 
el promedio general por carrera en los procesos de afiliación, punto que será interesante abordar en otra investigación.

Observación en el salón de clase

El tipo de observación para esta investigación fue del tipo participativa, a lo largo de las ocho semanas del proceso de observación; además, se debe considerar que el espacio que ocupó el personal docente en el salón de clase durante este periodo no fue siempre preferente, ya que en el proceso participaron dos docentes que alternaron su trabajo y participación.

Como resultado del trabajo de la observación en el salón de clase, se identifican y extraen, de las fichas de observación, algunas citas en los fragmentos de textos, que se pueden asociar a los modelos de afiliación propuestos.

En las tres primeras semanas de observación, se detecta una dinámica de agrupación similar en los grupos de trabajo, en donde el estudiantado se organiza, elige quien coordine o representante, y se distribuye funciones. Al sentirse cómodo, expresa libremente lo que quiere, lo que piensa, se facilita la comunicación, la confianza individual y grupal, y se logra, además, mayor efectividad en el trabajo grupal (Devine, 2002).

... y los otros se movilizan por el salón de clase, hay participación general y un ambiente des estresado, aparentemente tienen muchas ganas de iniciar su trabajo y se ven entusiasmados con los sistemas de votación, parece que se conocen desde siempre, se percibe un ambiente sin estrés (Ficha de observación 1, sección trabajo grupal, 25 de agosto del 2015).

... designan un representante para presentar el trabajo de grupo. Se distribuyeron las funciones, hay armonía en el ambiente, camaradería, ganas de trabajar (Ficha de observación 2, sección trabajo grupal, 1 de setiembre del 2015).

Al conformar grupos diferentes todas las semanas y al haber utilizado como medio de seguimiento el sistema de votación, se detectó que la coordinación grupal no siempre fue asumida por la misma persona, esto indica que el trabajo de grupos al azar propicia un liderazgo no jerárquico, un ambiente de inclusión en donde cada quien puede expresar sus ideas (Outcalt, et ali, 2001).

... siempre es una persona diferente la que lidera los equipos. Aparentemente se alterna el liderazgo (Ficha de observación 4, sección trabajo grupal, 22 de setiembre del 2015).

En los episodios correspondientes a las semanas 5 y 6, la participación aumentó, mejoró la interacción del estudiante, se percibió un gran espíritu de colaboración y compañerismo, entre estudiantes, confirmando que en el trabajo grupal, además de ser un recurso metodológico en donde el estudiantado aprende y enseña temas específicos, aprende en forma sistematizada (Pujolàs, 2009).

... hubo participación de todos los miembros de los grupo (Ficha de observación 5, sección trabajo grupal, 29 de setiembre del 2015).

... en la práctica grupal, aparentemente trabajan por igual, hay espíritu de colaboración y positivismo (Ficha de observación 6, sección trabajo grupal, día 6).

En los episodios 7 y 8 se trabajó con dos modalidades de conformación grupal, grupos impuestos por el personal docente y grupos al azar, esto se planificó con el propósito de seguir el comportamiento del estudiantado, en estas dos modalidades de trabajo en grupo, cuando ya tiene 6 semanas de conocerse. Un punto interesante a destacar en los siguientes episodios es la diferencia de comportamiento que presenta el estudiantado cuando el personal docente asigna los grupos y a la persona coordinadora, en cuyo caso disminuyó la interacción entre estudiantes y la mayor parte de responsabilidad la asume quien coordina cada grupo, por ejemplo:

... al asignar los grupos y el coordinador de grupo, es evidente un cambio en la dinámica de trabajo grupal, hay menos interacción entre los miembros del grupo se puede decir que nula los coordinadores asignan trabajo a los estudiantes....el coordinador presenta todos los resultados...no hay participación activa de los estudiantes....cambia la dinámica cuando de 
nuevo se retorna al sistema de conformación de grupos al azar, se retorna a la interacción entre estudiantes observada en los 6 primeros episodios (Ficha de observación 7, sección trabajo grupal, 6 de octubre del 2015).

En general, se encontró que el estudiantado delega mayor responsabilidad a quien coordina el grupo, cuando este rol es impuesto por el personal docente. Además, se puede decir que al cambiar la dinámica del trabajo grupal y, consecuentemente, la forma en que interacciona el estudiantado, la metodología empleada por el personal docente es la que define este punto, esto será fundamental para la consecución de las intenciones educativas (Madrid y Mayorga, 2012).

Entrevistas a docentes

El personal docente reconoce que en la carrera de Ingeniería Mecánica con Énfasis en Protección de Incendios no hay grupos de estudiantes que se mantengan, todos los grupos pueden trabajar indiferentemente con todos, comunicarse e interaccionar sin problema al respecto:

... todos se conocen, todos se tratan es decir más difícil ver núcleos consolidados de 5 o 6 compañeros sino todos se comunican con todos... (Entrevista docente 1, Líneas 16-19, p.3).

... yo no he visto que se armen grupos, tal vez por ser grupillos tan pequeños, todos trabajan siempre con todos (Entrevista docente3, líneas 27-28, p.1).

... los grupos no son siempre los mismos si se les pide formar grupos al azar, no veo que tengan problemas para cambiar de grupo como compañeros de trabajo (Entrevista docente 2, líneas 46-49, p.2).

Por otro lado, es evidente que la dinámica del trabajo grupal cambia la forma en que interactúa el estudiantado:

... si se trata de trabajo en grupos preestablecidos en donde se asigna un coordinador la dinámica de trabajo es muy diferente hay como menos interacción creo (Entrevista docente 4, líneas 19-20, p.2).

... pero cuando yo asigno grupo y coordinador de grupo es diferente, casi siempre el coordinador asignado es el que responde todo y delega todo (Entrevista docente 1, líneas 25-28, p.4).

De acuerdo con los fragmentos de los textos mostrados anteriormente, el personal docente afirma que el trabajo en grupos va a referir a estrategias didácticas con las que aborda las lecciones y que estos métodos definen la dinámica de trabajo de sus estudiantes. Además, cuando el sujeto docente asigna los grupos de trabajo y jerarquiza, nombrando a quien coordina, la interacción entre estudiantes disminuye, pues se respeta la jerarquía. Cabe destacar y de acuerdo con Alonso (2006), que el aula no es un elemento neutro: es, además de un espacio físico con elementos propios, un lugar en donde la guía docente va a interferir directamente con la forma en que sus estudiantes interaccionan y trabajan (Brunner, 1988).

Modelos de afiliación generados en la Sede Interuniversitaria de Alajuela

El análisis de datos lleva a proponer una clasificación básica para los modelos de afiliación que se dan en la Sede Interuniversitaria de Alajuela: el modelo de afiliación formal y el modelo de afiliación informal, cuyas características se presentan en la Tabla 2. 
TABLA 2

Características generales de los modelos de afiliación para estudiantes de la Sede Interuniversitaria de Alajuela

\begin{tabular}{|c|c|}
\hline $\begin{array}{l}\text { Modelo de afiliación } \\
\text { formal con enlace }\end{array}$ & $\begin{array}{l}\text { Modelo de afiliación } \\
\text { informal }\end{array}$ \\
\hline $\begin{array}{l}\text { Se imponen normas que } \\
\text { deben ser respetadas } \\
\text { por el individuo si se va } \\
\text { a integrar a } \\
\text { determinado grupo, se } \\
\text { impone una linea de } \\
\text { afiliación por medio de } \\
\text { diferentes procesos, no } \\
\text { hay libertad para elegir, } \\
\text { ni espacios para } \\
\text { resistirse. Hay } \\
\text { imposición en el proceso } \\
\text { de afiliación. }\end{array}$ & $\begin{array}{l}\text { Se permite al individuo } \\
\text { una mayor } \\
\text { espontaneidad, } \\
\text { creatividad, libertad, } \\
\text { juego, espacios de } \\
\text { resistencia y una } \\
\text { contracultura. No hay } \\
\text { imposición en el proceso } \\
\text { de afiliación. }\end{array}$ \\
\hline
\end{tabular}

Nota: Elaboración propia de la autora (2015).

Cada modelo de afiliación descrito en la Tabla 2 responde a diferentes procesos de enseñanza y de aprendizaje, dictados por las estrategias utilizadas por el personal docente en el salón de clase, durante el desarrollo de esta investigación. Estos procesos se presentan en el siguiente apartado.

Procesos de afiliación generados durante el trabajo grupal

Cuando el personal docente trabaja en grupos se destacan dos tipos de proceso de afiliación: el de comunicación por enlace vertical y el de comunicación por enlace horizontal (Girbal, Gardeña y Peñalva, 2012).

\section{Proceso de afiliación grupal de comunicación por enlace vertical}

Este proceso es característico del modelo de afiliación formal y se genera cuando los grupos de trabajo, las reglas, y quien coordina el grupo son impuestos por el personal docente. Se puede observar este proceso en la Figura 7. 


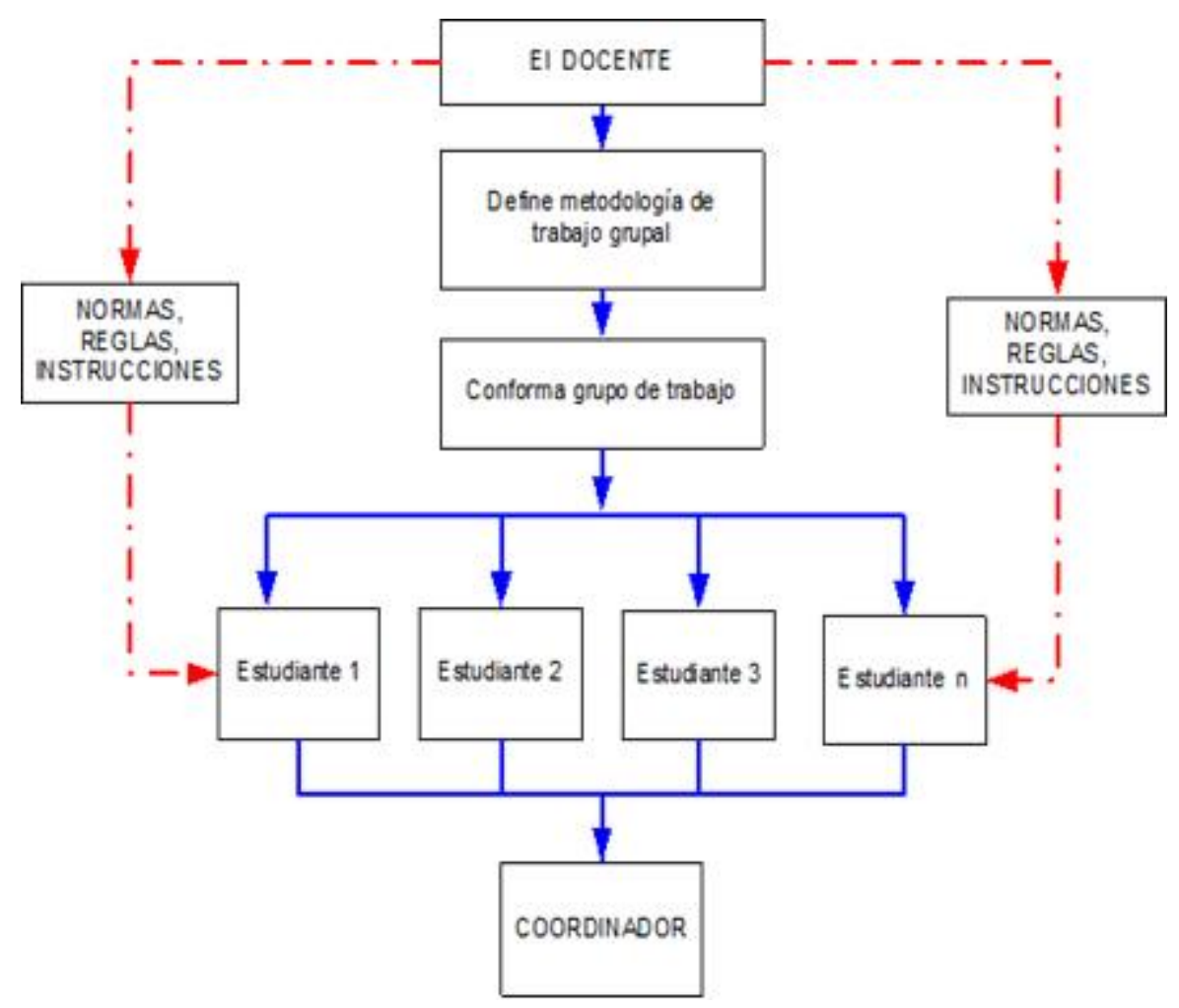

FIGURA 7.

Procesos de afiliación de grupal de enlace vertical de comunicación. Elaboración propia de la autora (2015).

\section{Proceso de afiliación grupal por comunicación por enlace horizontal}

Este proceso es característico del modelo informal de afiliación. Los enlaces que se generan en este proceso no son impuestos por el personal docente y pueden ser de corto, mediano o largo plazo. El estudiantado decide libremente con quién quiere trabajar y por cuánto tiempo. No se establece una jerarquía inmediata, la coordinación de acciones rota entre participantes. Se puede observar este proceso en la Figura 8. 


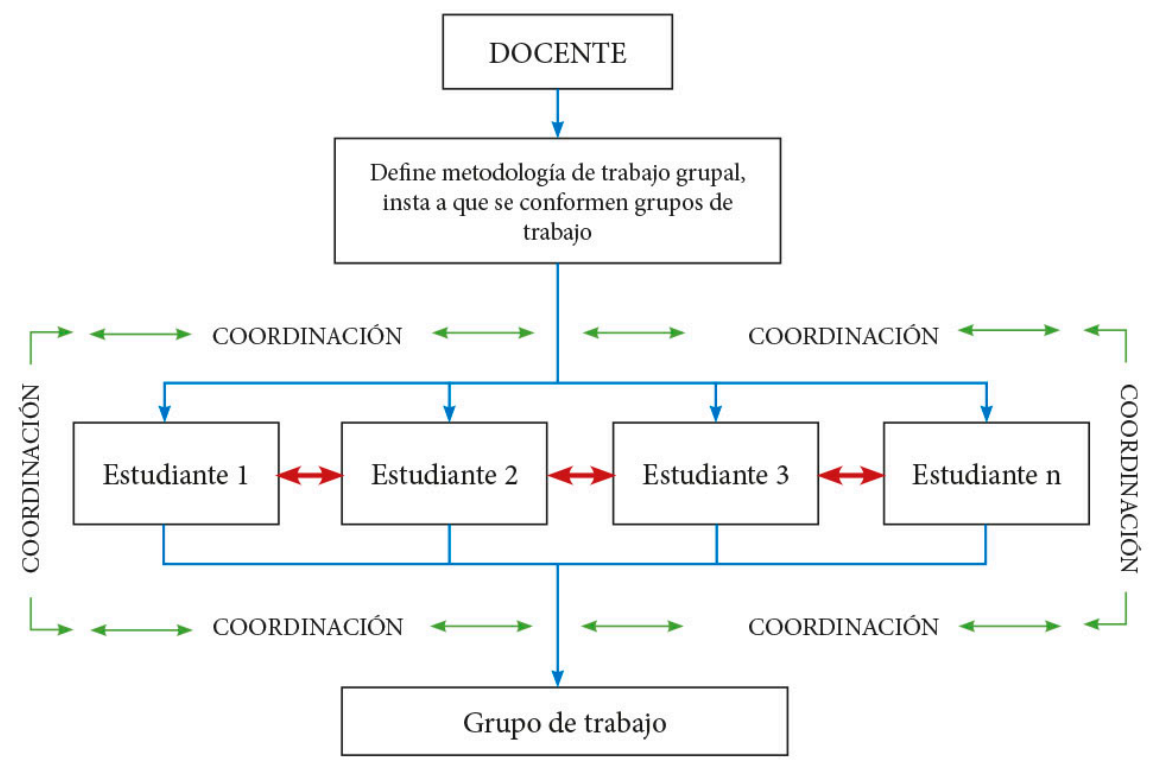

FIGURA 8

Proceso de afiliación grupal con encadenamiento horizontal.

Elaboración propia del autor (2015).

\section{Conclusiones}

Conocer la forma en que se afilia el estudiantado es una herramienta básica para el personal docente que lo guiará en los procesos de enseñanza y de aprendizaje que se generan en su salón de clase, ya que, solo el hecho de identificar los modelos de afiliación que sigue su población estudiantil, lo ayudará a definir las estrategias didácticas de trabajo, las cuales se pueden considerar base fundamental para la reformulación curricular. En este sentido, es importante destacar que en el proceso de investigación no se encontró reporte alguno de investigaciones sobre procesos de afiliación en los salones de clase.

En este trabajo se logró identificar dos clases de modelos de afiliación, generados cuando el personal docente aplica la metodología de trabajo grupal:

a) El modelo de afiliación informal, que se caracteriza especialmente por la interacción, cooperación, participación general de estudiantes en sus grupos de trabajo y los enlaces de comunicación horizontal. Este modelo propicia, en el individuo, mayor espontaneidad, creatividad, libertad, juego, espacios de resistencia y una contracultura, esto, al no imponerse normativas ni líneas definidas de afiliación.

b) El modelo de afiliación formal, que se caracteriza especialmente por la poca interacción entre estudiantes, participación casi nula en la resolución de problemas, delegación de responsabilidades en quien coordina el grupo de trabajo y enlaces de comunicación vertical claramente definidos. En este modelo el personal docente impone normas que deben de ser respetadas por el individuo al integrarse a un grupo de trabajo previamente determinado y, además una línea de afiliación con la que no se da al estudiante libertad para elegir, ni espacios para resistirse.

Una herramienta importante utilizada en la identificación de estos modelos fue el sistema de votación, que permitió identificar con mayor facilidad la forma como se asocian estudiantes, su participación grupal y general. Además, este sistema generó los datos necesarios para construir la matriz adyacente asimétrica en la que se reporta la frecuencia de no afiliación y que permite, así, el poder representar gráficamente los mapas o redes de afiliación general e individual, con ayuda del programa Net Draw. 
Por otro lado, en esta investigación emergen la capacidad de liderazgo y la capacidad de libertad, dimensiones que están asociadas a los modelos de afiliación caracterizados en la población, las cuales pueden ser objeto de estudio para futuras investigaciones, y que probablemente contribuirán a la conformación de nuevas estrategias didácticas para el desarrollo del trabajo grupal en clase.

Otros puntos interesantes a valorar para futuras investigaciones son: a) los modelos de afiliación que caracterizan a las estudiantes de Ingeniería, ya que en el transcurso de la investigación hubo evidencia de que siguen modelos diferentes, pero no se profundizó en este tema, ya que la evidencia se develó cuando se inició el análisis de resultados, b) el efecto de la ubicación, preferente y no preferente, de cada docente cuando se trabaja de forma grupal, puesto que se detectó que el comportamiento estudiantil puede cambiar dependiendo del espacio físico que ocupe su docente y c) la relación existente entre las prácticas socioeducativas transmitidas en las comunidades primarias, con el modelo de afiliación informal.

Es necesario e indispensable para el personal docente conocer los rasgos reales de la población con que trabaja: su contexto, su comunidad, sus capacidades; en fin, sus puntos de encuentro y desencuentro, ya que de esta forma podrá ir construyendo una metodología apropiada, que potencie los procesos de enseñanzaaprendizaje que se desarrollan en su salón de clase. Por otro lado, conocer las características de una población es una herramienta base para la formulación de una propuesta pedagógica, dado que esta debe orientar la estrategias y metodologías del proceso de enseñanza y de formación docente. Queda clara, de esta forma, la intencionalidad del proyecto educativo.

\section{REFERENCIAS}

Alonso, R. (2006). Contextos arquitectónicos del medio ambiente: De la arquitectura escolar a la del conocimiento. Observatorio medioambiental, 9, 267-296.

Aronowitz, S. y Giroux, H. A. (1985). Education Under Siege. London: Routledge.

Brunner, J. (1988). Realidad mental y mundos posibles. Barcelona: Gedisa.

Bourdieu, P. (1991). El sentido práctico. Madrid : Taurus.

Cejudo, R. (2006). Desarrollo humano y capacidades. Aplicaciones de la teoría de las capacidades de Amrtya Sen a la educación. Revista Española de Pedagogía, 2, 234-260.

Crotty, M. (1998). The Foundations of social research: Meaning and perspective in the research process. London: SAGE Publications.

Devine, D. (2002). A review and integration of classification systems relevant to teams in organizations. Group Dynamics: Theory. Research and Practice, 6(4), 291-310.

Forner, A. y Latorre, A. (1996). Diccionario terminológico de investigación educativa y psicopedagogía. Barcelona: EUB.

Gough,I. (2007). El enfoque de las capacidades de M. Nussbaum: Un análisis comparado con nuestra teoría de las necesidades humanas. Papeles de relaciones ecosociales y cambio global, 100. CIP.Ecosocial/Icaria.

Girbal, E. R., Gardella, C. H., y Peñalva, M. D. C. (2012). Proceso de Integración vertical y horizontal para contribuir a la excelencia académica de la carrera de Ingeniería en Sistemas de Información. Argentina: Universidad de la Plata.

Latorre, A., Del Rincón, D. y Arnal, J. (1997). Bases metodológicas de la investigación cualitativa. Barcelona: Hurtado.

Lozano, M. (2010). Modelos de asociatividad: Estrategias efectivas para el desarrollo de Pymes. Revista Escuela de Administración de Negocios, 68, 175-178. Recuperado de http://www.redalyc.org/articulo.oa? id $=20619844015$

Madrid, D. y Mayorga, M. (2012). La organización del espacio en el aula infantil. De las experiencias prácticas. Barcelona: Octadro. 
Hennia María Cavallini Solano. Un modelo de afiliación informal: Prácticas socioeducativas de estu...

Marchesi, A. (2000). Cambios sociales y educativos en Latinoamérica. Seminario sobre prospective educativa.UNESCO-Santiago de Chile. Recuperado de http://www.oei.es/reformaseducativas/ cambios_sociales_cambios_educativos_AL_marchesi.pdfenero del 2014

Nussbaum, M. (1992). Human functioning and social justice: in defense of Aristotelian essentialism, Polytical Theory, 2(22). 202-246.

Nussbaum, M. (2005). El cultivo de la humanidad. Una defensa clásica de la reforma en la educación liberal. Barcelona: Paidós Ibérica.

Outcalt, C., Faris, S., \& Mcmahon, K. (2001). Developing nonleadership on campus: Case studies and best practice in higher education. Westport, CT: Grenwood Press.

Pujolàs,P. (2009). Aprendizaje coorperativo y educación inclusiva: Una forma práctica de aprender juntos alumnos diferentes. VI. Jornadas de cooperación educativa en Iberoamérica. Antigua Guatemala.

Sen, A. (1981). Poverty and famines. Oxford: Oxford University Press.

Sen, A. (1999). Desarrollo y libertad. Buenos Aires: Edorial Planeta.

Telle, M. (2004). Estudio de clusters y desarrollo regional: Casos de Piura y Loreto. Perú: Consorcio de Investigaciones Económicas.

Zamudio, S. (2011). La afiliación y el aprovechamiento escolar: Los estudiantes de pedagogía de la UPN (Tesis de Licenciatura). Universidad Pedagógica Nacional de México.

\section{Notas}

[2] Habitus, sistemas de disposiciones duraderas y transferibles, estructuradas predispuestas para funcionar como estructurantes.......como principios generadores y organizadores de prácticas y representaciones que pueden estar objetivamente adaptadas a su fin sin suponer la búsqueda consciente de fines y el dominio expreso de las operaciones necesarias para alcanzarlos, objetivamente $<<$ reguladas $>>$ y $<<$ regulares $>>$ sin ser el producto de la obediencia de las reglas, y, a la vez que todo esto, colectivamente orquestadas sin ser el producto de la acción organizadora de un director de orquesta (Bourdieu, 1991, p. 92)

[3] Campus, Según Bourdieu (1991), campo social, constituido por una estructura de relaciones, es una construcción histórica y social, un espacio de juego conformado por instituciones específicas que tienen sus propias leyes de funcionamiento

\section{BY-NC-ND}

